\title{
Existence and uniqueness of an inverse problem for a second order hyperbolic equation
}

\author{
İbrahim Tekin \\ Department of Mathematics, Bursa Technical University, Yıldırım-Bursa, 16310, Turkey \\ Corresponding authorE-mail: ibrahim.tekin@btu.edu.tr
}

\author{
Article Info \\ Keywords: Second order hyperbolic \\ equation, Inverse problem, Finite dif- \\ ference method. \\ 2010 AMS: 35R30, 35L10, 65M06 \\ Received: 2 July 2018 \\ Accepted: 16 August 2018 \\ Available online: 30 September 2018
}

Abstract

In this paper, an initial boundary value problem for a second order hyperbolic equation is considered. Giving an additional condition, a time-dependent coefficient multiplying a linear term is determined and existence and uniqueness theorem for small times is proved. The finite difference method is proposed for solving the inverse problem numerically.

\section{Introduction and problem formulation}

Many physical models include unknown coefficients in the equation and the solution of the inverse problems consisting of the identification of these coefficients has become a very popular area of research in recent years. The inverse problems for hyperbolic equations present significant value for physical applications such as vibrating string, seismology, geophysics etc.

Consider the problem for the vibrating string with arbitrary initial conditions

$$
\begin{aligned}
& v_{t t}=c^{2} v_{x x}+a(t) v(x, t)+s(x, t), \quad(x, t) \in D_{T}, \\
& v(x, 0)=v_{0}(x), v_{t}(x, 0)=v_{1}(x), 0 \leq x \leq 1,
\end{aligned}
$$

where $D_{T}=\{(x, t): 0<x<1,0 \leq t \leq T\}$ for some fixed $T>0, c=\frac{\Upsilon}{\rho}$ known as phase velocity of the wave motion, $\Upsilon$ is the force of tension exerted on the string, $\rho$ is the mass of density, $s(x, t)$ is external forcing function, $v=v(x, t)$ represents the wave displacement at position $x$ and time $t$ and the functions $v_{0}(x)$ and $v_{1}(x)$ are wave modes or kinks and velocity, respectively. In this paper, we will get $c=1$ for simplicity.

For a given function $a(t)$, the problem of finding $v(x, t)$ from the equation (1.1) with the initial condition (1.2) and the boundary condition

$$
v(0, t)=b(t), v_{x}(0, t)=v_{x}(1, t), 0 \leq t \leq T,
$$

is called direct (forward) problem. The boundary condition $v(0, t)=b(t)$ occurs if the left end of string is attached to a spring-mass system and $b(t)$ is the position of the mass at the left end.

If $a(t)$ is unknown, finding the pair of solution $\{a(t), v(x, t)\}$ of the problem (1.1)-(1.3) with the additional condition

$$
v(1, t)=r(t), 0 \leq t \leq T
$$


is called inverse problem.

The inverse problems for the hyperbolic equation with different boundary conditions and space dependent coefficients are studied in [7, 10 , $14,16]$ and more recently in $[5,15]$. The inverse problem for the hyperbolic equation with time dependent coefficient with integral condition is investigated in [11].

For the some numerical aspects of initial and initial-boundary value problems of the hyperbolic equations is considered for direct problem in [3], for finding unknown source term in [2,9], for finding space dependent potential in [1, 4] and for finding time dependent source function of time-fractional wave equation in [17].

The article is organized as following: In Section 2, we rewrite the problem with homogeneous boundary conditions by a simple transformation and present auxiliary spectral problem of this problem and its properties. In Section 3, the series expansion method in terms of eigenfunctions converts the new inverse problem to a fixed point problem in a suitable Banach space. Under some consistency, regularity conditions on initial and boundary data the existence and uniqueness of the solution of the inverse problem is shown by the way that the fixed point problem has unique solution for small $T$. In Section 4, we solve the inverse problem numerically by applying finite difference method. We also present numerical example to illustrate the behaviour of the proposed method.

\section{Auxiliary spectral problem}

Since the boundary condition (1.3) is non-homogeneous, we introduce a new variable $u(x, t)=v(x, t)-b(t)$. Then, from Eqs.(1.1)-(1.4), it is easy to see that $u(x, t)$ satisfies the following problem:

$$
\begin{aligned}
& u_{t t}=u_{x x}+a(t) u(x, t)+f(x, t), \quad(x, t) \in D_{T}, \\
& u(x, 0)=\varphi(x), u_{t}(x, 0)=\psi(x), 0 \leq x \leq 1, \\
& u(0, t)=0, u_{x}(0, t)=u_{x}(1, t), 0 \leq t \leq T, \\
& u(1, t)=h(t), 0 \leq t \leq T,
\end{aligned}
$$

where $f(x, t)=s(x, t)+a(t) b(t)-b^{\prime \prime}(t), \varphi(x)=v_{0}(x)-b(0), \psi(x)=v_{1}(x)-b^{\prime}(0)$ and $h(t)=r(t)-b(t)$.

We attempt to apply the Fourier method of eigenfunction expansion to the problem (2.1)-(2.4). Auxiliary spectral problem of the problem (2.1)-(2.3) is

$$
\begin{gathered}
X^{\prime \prime}(x)+\lambda X(x)=0,0 \leq x \leq 1, \\
X(0)=0, X^{\prime}(0)=X^{\prime}(1) .
\end{gathered}
$$

The problem (2.5) has eigenvalues $\lambda_{k}=\left(\mu_{k}\right)^{2}=(2 \pi k)^{2}, k=0,1,2, \ldots$ and corresponding eigenfunctions

$$
X_{0}(x)=x, X_{2 k-1}(x)=x \cos \mu_{k} x, X_{2 k}(x)=\sin \mu_{k} x, k=1,2, \ldots .
$$

It is known from [6] that the spectral problem (2.5) is not self-adjoint. Then we have to consider the adjoint spectral problem. The adjoint spectral problem of $(2.5)$ is

$$
\begin{gathered}
Y^{\prime \prime}(x)+\lambda Y(x)=0,0 \leq x \leq 1, \\
Y^{\prime}(1)=0, Y(0)=Y(1) .
\end{gathered}
$$

This problem has the same eigenvalues as in the problem (2.5) and corresponding eigenfunctions

$$
Y_{0}(x)=2, Y_{2 k-1}(x)=4 \cos \mu_{k} x, Y_{2 k}(x)=4(1-x) \sin \mu_{k} x, k=1,2, \ldots
$$

The systems (2.6) and (2.8) arise in [6] for the solution of non-local boundary value problem in heat equation. It is easy to verify that the systems (2.6) and (2.8) are bi-orthonormal on $[0,1]$, i.e.

$$
\left(X_{i}(x), Y_{j}(x)\right)=\int_{0}^{1} X_{i}(x) Y_{j}(x) d x=\left\{\begin{array}{c}
0, i \neq j \\
1, i=j
\end{array}\right.
$$

Moreover the system (2.6) forms a basis in $L_{2}[0,1]$ and they are also Riesz basis in $L_{2}[0,1]$. Then any function $g(x) \in L_{2}[0,1]$ is expanded in bi-orthogonal series

$$
g(x)=\sum_{k=0}^{\infty} g_{k} X_{k}(x)
$$


where $g_{k}=\int_{0}^{1} g(x) Y_{k}(x) d x, k=0,1,2, \ldots$ and the estimate

$$
r\|g(x)\|_{L_{2}[0,1]}^{2} \leq \sum_{k=0}^{\infty} g_{k}^{2} \leq R\|g(x)\|_{L_{2}[0,1]}^{2}
$$

is valid for any function $g(x) \in L_{2}[0,1]$, here $r=3 / 4, R=16$.

Let us introduce the functional space [12]

$$
\begin{aligned}
B_{2, T}^{3} & =\left\{u(x, t)=\sum_{k=0}^{\infty} u_{k}(t) X_{k}(x): u_{k}(t) \in C[0, T], J_{T}(u)=\left\|u_{o}(t)\right\|_{C[0, T]}\right. \\
& \left.+\left(\sum_{k=1}^{\infty}\left(\mu_{k}^{3}\left\|u_{2 k}(t)\right\|_{C[0, T]}\right)^{2}\right)^{1 / 2}+\left(\sum_{k=1}^{\infty}\left(\mu_{k}^{3}\left\|u_{2 k-1}(t)\right\|_{C[0, T]}\right)^{2}\right)^{1 / 2}<+\infty\right\},
\end{aligned}
$$

with the norm $\|u(x, t)\|_{B_{2, T}^{3}} \equiv J_{T}(u)$ which is related with the Fourier coefficients of the function $u(x, t)$ by the eigenfunctions $X_{k}(x)$, $k=0,1,2, \ldots$. It is shown in [8] that $B_{2, T}^{3}$ is Banach space. Obviously $E_{T}^{3}=B_{2, T}^{3} \times C[0, T]$ of the vector function $z(x, t)=\{a(t), u(x, t)\}$ with the norm $\|z(x, t)\|_{E_{T}^{3}}=\|a(t)\|_{C[0, T]}+\|u(x, t)\|_{B_{2, T}^{3}}$ is also Banach space.

\section{Existence and uniqueness of the solution}

The pair $\{a(t), u(x, t)\}$ from the class $C[0, T] \times C^{2}\left(\bar{D}_{T}\right)$ for which the conditions (2.1)-(2.4) are satisfied, is called a classical solution of the inverse problem (2.1)-(2.4).

Since the system (2.6) forms Riesz basis and the systems (2.6),(2.8) are bi-orthogonal in $L_{2}[0,1]$ and the function $a(t)$ is time dependent, seeking the solution of the problem (2.1)-(2.4) in the following form is suitable:

$$
u(x, t)=\sum_{k=0}^{\infty} u_{k}(t) X_{k}(x),
$$

where $u_{k}(t)=\int_{0}^{1} u(x, t) Y_{k}(x) d x, k=0,1,2, \ldots$

For an arbitrary $a(t) \in C[0, T]$, the solution of the problem (2.1)-(2.4) can be written as

$$
u(x, t)=u_{0}(t) X_{0}(x)+\sum_{k=1}^{\infty} u_{2 k-1}(t) X_{2 k-1}(x)+\sum_{k=1}^{\infty} u_{2 k}(t) X_{2 k}(x) .
$$

By using the Fourier's method, it is easy to obtain that $u_{k}(t), k=0,1,2, \ldots$ should be satisfies the equations:

$$
u_{0}^{\prime \prime}(t)=a(t) u_{0}(t)+f_{0}(t),
$$

$$
u_{2 k-1}^{\prime \prime}(t)+\mu_{k}^{2} u_{2 k-1}(t)=a(t) u_{2 k-1}(t)+f_{2 k-1}(t), k=1,2, \ldots,
$$

$$
u_{2 k}^{\prime \prime}(t)+\mu_{k}^{2} u_{2 k}(t)=a(t) u_{2 k}(t)+f_{2 k}(t)-2 \mu_{k} u_{2 k-1}(t), k=1,2, \ldots
$$

$$
u_{k}(0)=\varphi_{k}, \quad u_{k}^{\prime}(0)=\psi_{k}, k=0,1,2, \ldots,
$$

where $f_{k}(t)=\int_{0}^{1} f(x, t) Y_{k}(x) d x, \varphi_{k}=\int_{0}^{1} \varphi(x) Y_{k}(x) d x, \psi_{k}=\int_{0}^{1} \psi(x) Y_{k}(x) d x, k=0,1,2, \ldots$ Solving the problem (3.2)-(3.5), we obtain

$$
\begin{aligned}
& u_{0}(t)=\varphi_{0}+t \psi_{0}+\int_{0}^{t}(t-\tau) F_{0}(\tau ; u, a) d \tau, 0 \leq t \leq T, \\
& u_{2 k-1}(t)=\varphi_{2 k-1} \cos \mu_{k} t+\frac{1}{\mu_{k}} \psi_{2 k-1} \sin \mu_{k} t+\frac{1}{\mu_{k}} \int_{0}^{t} F_{2 k-1}(\tau ; u, a) \sin \mu_{k}(t-\tau) d \tau, k=1,2, \ldots, \\
& u_{2 k}(t)=\varphi_{2 k} \cos \mu_{k} t+\frac{1}{\mu_{k}} \psi_{2 k} \sin \mu_{k} t+\frac{1}{\mu_{k}} \int_{0}^{t} F_{2 k}(\tau ; u, a) \sin \mu_{k}(t-\tau) d \tau \\
& \quad-t \varphi_{2 k-1} \sin \mu_{k} t-\frac{1}{\mu_{k}} \psi_{2 k-1}\left[\frac{1}{\mu_{k}} \sin \mu_{k} t-t \cos \mu_{k} t\right] \\
& \quad-\frac{2}{\mu_{k}} \int_{0}^{t} \int_{0}^{\tau} F_{2 k-1}(\xi ; u, a) \sin \mu_{k}(\tau-\xi) d \xi \sin \mu_{k}(t-\tau) d \tau, k=1,2, \ldots,
\end{aligned}
$$

where $F_{k}(t ; u, a)=a(t) u_{k}(t)+f_{k}(t), k=0,1,2, \ldots$ 
Substituting (3.6)-(3.8) into (3.1),

$$
\begin{aligned}
& u(x, t)=\left(\varphi_{0}+t \psi_{0}+\int_{0}^{t}(t-\tau) F_{0}(\tau ; u, a) d \tau\right) X_{0}(x) \\
& +\sum_{k=1}^{\infty}\left(\varphi_{2 k-1} \cos \mu_{k} t+\frac{1}{\mu_{k}} \psi_{2 k-1} \sin \mu_{k} t+\frac{1}{\mu_{k}} \int_{0}^{t} F_{2 k-1}(\tau ; u, a) \sin \mu_{k}(t-\tau) d \tau\right) X_{2 k-1}(x) \\
& +\sum_{k=1}^{\infty}\left(\varphi_{2 k} \cos \mu_{k} t+\frac{1}{\mu_{k}} \psi_{2 k} \sin \mu_{k} t+\frac{1}{\mu_{k}} \int_{0}^{t} F_{2 k}(\tau ; u, a) \sin \mu_{k}(t-\tau) d \tau-t \varphi_{2 k-1} \sin \mu_{k} t\right. \\
& \left.-\frac{1}{\mu_{k}} \psi_{2 k-1}\left[\frac{1}{\mu_{k}} \sin \mu_{k} t-t \cos \mu_{k} t\right]-\frac{2}{\mu_{k}} \int_{0}^{t} \int_{0}^{\tau} F_{2 k-1}(\xi ; u, a) \sin \mu_{k}(\tau-\xi) d \xi \sin \mu_{k}(t-\tau) d \tau\right) X_{2 k}(x) .
\end{aligned}
$$

Consider $x=1$ in the equation (2.1) and by using the over-determination condition (2.4), we obtain

$$
a(t)=\frac{1}{h(t)}\left[h^{\prime \prime}(t)-f(1, t)+\sum_{k=1}^{\infty} \mu_{k}^{2}\left(\varphi_{2 k-1} \cos \mu_{k} t+\frac{\psi_{2 k-1}}{\mu_{k}} \sin \mu_{k} t+\frac{1}{\mu_{k}} \int_{0}^{t} F_{2 k-1}(\tau ; u, a) \sin \mu_{k}(t-\tau) d \tau\right)\right] .
$$

Thus, we get the equalities of the pair $\{a(t), u(x, t)\}$.

Let us denote $z=[a(t), u(x, t)]^{T}$ and consider the operator equation

$$
z=\Phi(z)
$$

The operator $\Phi$ is determined in the set of functions $z$ and has the form $\left[\phi_{0}, \phi_{1}\right]^{T}$, where

$$
\begin{aligned}
& \phi_{0}(z)=\frac{1}{h(t)}\left[h^{\prime \prime}(t)-f(1, t)+\sum_{k=1}^{\infty} \mu_{k}^{2}\left(\varphi_{2 k-1} \cos \mu_{k} t+\frac{\psi_{2 k-1}}{\mu_{k}} \sin \mu_{k} t+\frac{1}{\mu_{k}} \int_{0}^{t} F_{2 k-1}(\tau ; u, a) \sin \mu_{k}(t-\tau) d \tau\right)\right], \\
& \phi_{1}(z)=\left(\varphi_{0}+t \psi_{0}+\int_{0}^{t}(t-\tau) F_{0}(\tau ; u, a) d \tau\right) X_{0}(x) \\
& +\sum_{k=1}^{\infty}\left(\varphi_{2 k-1} \cos \mu_{k} t+\frac{1}{\mu_{k}} \psi_{2 k-1} \sin \mu_{k} t+\frac{1}{\mu_{k}} \int_{0}^{t} F_{2 k-1}(\tau ; u, a) \sin \mu_{k}(t-\tau) d \tau\right) X_{2 k-1}(x) \\
& +\sum_{k=1}^{\infty}\left(\varphi_{2 k} \cos \mu_{k} t+\frac{1}{\mu_{k}} \psi_{2 k} \sin \mu_{k} t+\frac{1}{\mu_{k}} \int_{0}^{t} F_{2 k}(\tau ; u, a) \sin \mu_{k}(t-\tau) d \tau-t \varphi_{2 k-1} \sin \mu_{k} t\right. \\
& \left.-\frac{1}{\mu_{k}} \psi_{2 k-1}\left[\frac{1}{\mu_{k}} \sin \mu_{k} t-t \cos \mu_{k} t\right]-\frac{2}{\mu_{k}} \int_{0}^{t} \int_{0}^{\tau} F_{2 k-1}(\xi ; u, a) \sin \mu_{k}(\tau-\xi) d \xi \sin \mu_{k}(t-\tau) d \tau\right) X_{2 k}(x) .
\end{aligned}
$$

Let us demonstrate that $\Phi$ maps $E_{T}^{3}$ onto itself continuously. In other words, we need to show $\phi_{0}(z) \in C[0, T]$ and $\phi_{1}(z) \in B_{2, T}^{3}$ for arbitrary $z=[a(t), u(x, t)]^{T}$ with $a(t) \in C[0, T], u(x, t) \in B_{2, T}^{3}$.

We will use the following assumptions on the data of problem (2.1) - (2.4):

$\left(A_{1}\right) \varphi(x) \in C^{3}[0,1], \varphi(0)=\varphi^{\prime \prime}(0)=0, \varphi^{\prime}(0)=\varphi^{\prime}(1)$

$\left(A_{2}\right) \psi(x) \in C^{2}[0,1], \psi(0)=0, \psi^{\prime}(0)=\psi^{\prime}(1)$,

$\left(A_{3}\right) h(t) \in C^{2}[0, T], h(0)=\varphi(1), h^{\prime}(0)=\psi(1)$,

(A $\left.A_{4}\right) f(x, t) \in C\left(\bar{D}_{T}\right), f_{x}, f_{x x} \in C[0,1], \forall t \in[0, T], f(0, t)=0, f_{x}(0, t)=f_{x}(1, t)$.

First, let us show that $\phi_{0}(z) \in C[0, T]$. Under the assumptions $\left(\mathrm{A}_{1}\right)-\left(\mathrm{A}_{4}\right)$, we obtain from (3.12)

$$
\left|\phi_{0}(z)\right| \leq \frac{1}{|h(t)|}\left[\left|h^{\prime \prime}(t)\right|+|f(1, t)|+\sum_{k=1}^{\infty}\left(\frac{1}{\mu_{k}}\left|\alpha_{2 k-1}\right|+\frac{1}{\mu_{k}}\left|\beta_{2 k-1}\right|+\frac{1}{\mu_{k}} \int_{0}^{T}\left[\left|\gamma_{2 k-1}(t)\right|+\mu_{k}^{2}|a(t)|\left|u_{2 k-1}(t)\right|\right] d t\right)\right],
$$

where $\varphi_{2 k-1}=\frac{1}{\mu_{k}^{3}} \alpha_{2 k-1}, \psi_{2 k-1}=\frac{1}{\mu_{k}^{2}} \beta_{2 k-1}, f_{2 k-1}(t)=\frac{1}{\mu_{k}^{2}} \gamma_{2 k-1}(t), \alpha_{2 k-1}=-4 \int_{0}^{1} \varphi^{\prime \prime \prime}(x) \sin \left(\mu_{k} x\right) d x, \beta_{2 k-1}=4 \int_{0}^{1} \psi^{\prime \prime}(x) \sin \left(\mu_{k} x\right) d x$, $\gamma_{2 k-1}(t)=4 \int_{0}^{1} f_{x x}(x, t) \sin \left(\mu_{k} x\right) d x$.

Using Cauchy-Schwartz and Bessel inequalities, we derive from the last inequality

$$
\left\|\phi_{0}(t)\right\|_{C[0, T]} \leq R_{1}(T)+R_{2}(T)\|a(t)\|_{C[0, T]}\|u(x, t)\|_{B_{2, T}^{3}},
$$


where $R_{1}(T)=\frac{1}{\|h(t)\|_{C[0, T]}}\left(\left\|h^{\prime \prime}(t)\right\|_{C[0, T]}+\|f(1, t)\|_{C[0, T]}+C_{0}\left(\left\|\varphi^{\prime \prime \prime}(x)\right\|_{L_{2}[0,1]}+\left\|\psi^{\prime \prime}(x)\right\|_{L_{2}[0,1]}+T\left\|f_{x x}(x, t)\right\|_{L_{2}\left(D_{T}\right)}\right)\right), R_{2}(T)=\frac{2 C_{1} T}{\|h(t)\|_{C[0, T]}}$, $C_{0}=\left(\sum_{k=1}^{\infty} \frac{1}{\mu_{k}^{2}}\right)^{1 / 2}, C_{1}=\left(\sum_{k=1}^{\infty} \frac{1}{\mu_{k}^{4}}\right)^{1 / 2}$. Thus $\phi_{0}(z)$ is continuous in $[0, T]$.

Now, let us verify that $\phi_{1}(z) \in B_{2, T}^{3}$, i.e.

$$
J_{T}\left(\phi_{1}\right)=\left\|\phi_{1,0}(t)\right\|_{C[0, T]}+\left(\sum_{k=1}^{\infty}\left(\mu_{k}^{3}\left\|\phi_{1,2 k}(t)\right\|_{C[0, T]}\right)^{2}\right)^{1 / 2}+\left(\sum_{k=1}^{\infty}\left(\mu_{k}^{3}\left\|\phi_{1,2 k-1}(t)\right\|_{C[0, T]}\right)^{2}\right)^{1 / 2}<+\infty,
$$

where $\phi_{1,0}(t), \phi_{1,2 k}(t)$ and $\phi_{1,2 k-1}(t)$ are the equal to the right hand side of $u_{0}(t), u_{2 k}(t)$ and $u_{2 k-1}$ as in (3.6)-(3.8), respectively. After some manipulations under the assumptions $\left(\mathrm{A}_{1}\right)-\left(\mathrm{A}_{4}\right)$,

$$
\left\|\phi_{1}\right\|_{B_{2, T}^{3}} \leq \widetilde{R}_{1}(T)+\widetilde{R}_{2}(T)\|a(t)\|_{C[0, T]}\|u(x, t)\|_{B_{2, T}^{3}},
$$

where $\widetilde{R}_{1}(T)=\|\varphi(x)\|_{C[0,1]}+T\|\psi(x)\|_{C[0,1]}+2 T^{3 / 2}\|f(x, t)\|_{C\left(D_{T}\right)}+(\sqrt{2}+4 T)\left\|\varphi^{\prime \prime \prime}(x)\right\|_{L_{2}[0,1]}+(4+4 T)\left\|\psi^{\prime \prime}(x)\right\|_{L_{2}[0,1]}$ $+\left(2 \sqrt{2 T}+8 T^{3 / 2}\right)\left\|f_{x x}(x, t)\right\|_{L_{2}\left(D_{T}\right)}+2\left\|\varphi^{\prime \prime \prime}(x)(1-x)-3 \varphi^{\prime \prime}(x)\right\|_{L_{2}[0,1]}+2\left\|\psi^{\prime \prime}(x)(1-x)-2 \psi^{\prime}(x)\right\|_{L_{2}[0,1]}+2 T^{1 / 2}\left\|f_{x x}(x, t)(1-x)-2 f_{x}(x, t)\right\|_{L_{2}\left(D_{T}\right)}$, $\widetilde{R}_{2}(T)=\max \left(T^{2}, 2 C_{0} T, 4 \sqrt{2} C_{0} T^{2}\right)$.

Since $J_{T}\left(\phi_{1}\right)<+\infty, \phi_{1}$ is belongs to the space $B_{2, T}^{3}$. Now, let $z_{1}$ and $z_{2}$ be any two elements of $E_{T}^{3}$. We know that $\left\|\Phi\left(z_{1}\right)-\Phi\left(z_{2}\right)\right\|_{E_{T}^{3}}=$ $\left\|\phi_{0}\left(z_{1}\right)-\phi_{0}\left(z_{2}\right)\right\|_{C[0, T]}+\left\|\phi_{1}\left(z_{1}\right)-\phi_{2}\left(z_{2}\right)\right\|_{B_{2, T}^{3}}$. Here $z_{i}=\left[a^{i}(t), u^{i}(x, t)\right]^{T}, i=1,2$.

Under the assumptions $\left(\mathrm{A}_{1}\right)-\left(\mathrm{A}_{4}\right)$, we obtain

$$
\left\|\Phi\left(z_{1}\right)-\Phi\left(z_{2}\right)\right\|_{E_{T}^{3}} \leq A(T) C\left(a^{1}, u^{2}\right)\left\|z_{1}-z_{2}\right\|_{E_{T}^{3}},
$$

where $A(T)=R_{2}(T)+\widetilde{R}_{2}(T)$ and $C\left(a^{1}, u^{2}\right)$ is the constant includes the norms of $\left\|a^{1}(t)\right\|_{C[0, T]}$ and $\left\|u^{2}(x, t)\right\|_{B_{2, T}^{3}}$.

For sufficiently small $T, 0<A(T)<1$. This implies that the operator $\Phi$ is contraction mapping which maps $E_{T}^{3}$ onto itself continuously. Then according to Banach fixed point theorem there exists a unique solution of (3.11).

Thus, we proved the following theorem:

Theorem 3.1. Let the assumptions $\left(A_{1}\right)-\left(A_{4}\right)$ be satisfied. Then, the inverse problem (2.1)-(2.4) has unique solution for small T.

Note that $s(x, t)=f(x, t)-a(t) b(t)-b^{\prime \prime}(t), v_{0}(x)=\varphi(x)+b(0), v_{1}(x)=\psi(x)+b^{\prime}(0)$ and $r(t)=h(t)+b(t)$ then under the following assumptions:

$\left(\widetilde{A}_{1}\right) v_{0}(x) \in C^{3}[0,1], v_{0}(0)=b(0), v_{0}^{\prime \prime}(0)=0, v_{0}^{\prime}(0)=v_{0}^{\prime}(1)$

$\left(\widetilde{A}_{2}\right) v_{1}(x) \in C^{2}[0,1], v_{1}(0)=b^{\prime}(0), v_{1}^{\prime}(0)=v_{1}^{\prime}(1)$,

$\left(\widetilde{A}_{3}\right) r(t) \in C^{2}[0, T], r(0)=v_{0}(1), r^{\prime}(0)=v_{1}(1)$

$\left(\widetilde{A}_{4}\right) s(x, t) \in C\left(\bar{D}_{T}\right), s_{x}, s_{x x} \in C[0,1], \forall t \in[0, T], s(0, t)=-a(t) b(t)+b^{\prime \prime}(t), s_{x}(0, t)=s_{x}(1, t)$,

the problem (1.1)-(1.4) has a unique classical solution $\{r(t), v(x, t)\}$ for small $T$ where $v(x, t)=u(x, t)+b(t)$.

\section{Numerical solution of the problem}

In this section,we describe the numerical method applied to the inverse initial boundary value problem (1.1)-(1.4). The discrete form of our problem is as follows: We divide the domain $(0,1) \times(0, T)$ into $n x$ and $n t$ subintervals of equal length $h x$ and $h t$, where $h x=1 / n x$ and $h t=T / n t$, respectively. We denote by $V_{j}^{n}:=V\left(x_{j}, t_{n}\right), a^{n}:=a\left(t_{n}\right)$ and $s_{j}^{n}:=s\left(x_{j}, t_{n}\right)$, where $x_{j}=j h x, t_{n}=n h t$ for $j=0, \ldots, n x, n=0, \ldots, n t$.

Then, a central difference approximation to the equations (1.1)-(1.3) at the mesh points $\left(x_{j}, t_{n}\right)$ is

$$
\begin{aligned}
& V_{j}^{n+1}=k^{2} V_{j+1}^{n}+2\left(1-k^{2}\right) V_{j}^{n}+k^{2} V_{j-1}^{n}-V_{j}^{n-1}+(h t)^{2}\left(a^{n} V_{j}^{n}+s_{j}^{n}\right), j=1, \ldots, n x-1, n=1, \ldots, n t-1, \\
& V_{j}^{0}=\left(v_{0}\right)_{j}, \quad j=0, \ldots, n x, \frac{V_{j}^{1}-V_{j}^{-1}}{2 h t}=\left(v_{1}\right)_{j}, j=1, \ldots, n x-1, \\
& V_{0}^{n}=b^{n}, \frac{V_{n x}^{n}-V_{n x-1}^{n}}{h x}=\frac{V_{1}^{n}-V_{0}^{n}}{h x}, n=0, \ldots, n t,
\end{aligned}
$$

where $k=\frac{h t}{h x}$. Equation (4.1) represents an explicit finite difference method which is stable for $k \leq 1$. Putting $n=0$ in the equation (4.1) and using (4.2), we obtain

$$
V_{j}^{1}=\frac{1}{2}\left(k^{2}\left(v_{0}\right)_{j+1}+2\left(1-k^{2}\right)\left(v_{0}\right)_{j}+k^{2}\left(v_{0}\right)_{j-1}+2 h t\left(v_{1}\right)_{j}+(h t)^{2}\left(a^{0}\left(v_{0}\right)_{j}+s_{j}^{0}\right)\right), j=1, \ldots, n x-1
$$


Consider (1.4) in the equation (1.1) at $x=1$, we obtain

$$
a(t)=\frac{r^{\prime \prime}(t)-v_{x x}(1, t)-s(1, t)}{r(t)} .
$$

After discretizing last equation, we have

$$
\begin{aligned}
& a^{n}=\frac{\left(r^{n+1}-2 r^{n}+r^{n-1}\right) /(h t)^{2}-\left(V_{n x}^{n}-2 V_{n x-1}^{n}+V_{n x-2}^{n}\right) /(h x)^{2}-s_{n x}^{n}}{r^{n}}, n=1, \ldots, n t-1, \\
& a^{n t}=\frac{\left(r^{n t}-2 r^{n t-1}+r^{n t-2}\right) /(h t)^{2}-\left(V_{n x}^{n t}-2 V_{n x-1}^{n t}+V_{n x-2}^{n t}\right) /(h x)^{2}-s_{n x}^{n t}}{r^{n t}}, \\
& a^{0}=\frac{\left(r^{2}-2 r^{1}+r^{0}\right) /(h t)^{2}-\left(V_{n x}^{0}-2 V_{n x-1}^{0}+V_{n x-2}^{0}\right) /(h x)^{2}-s_{n x}^{0}}{r^{0}} .
\end{aligned}
$$

Now let us consider (4.5)-(4.7) in (4.1), we obtain the system with respect to $V_{j}^{n}, j=0, \ldots, n x, n=0, \ldots, n t$ which can be solved explicitly. Then using the calculated values of $V_{n x-2}^{n}$ in (4.5)-(4.7), we obtain the values of $a^{n}, n=0, \ldots, n t$.

Example 4.1. Consider the inverse problem (1.1)-(1.4) with the input data

$$
\begin{aligned}
s(x, t) & =(1+2 \pi x-\sin 2 \pi x) \exp (t)-1-2 \pi x+\sin 2 \pi x, \\
v_{0}(x) & =1+2 \pi x-\sin 2 \pi x, v_{1}(x)=1+2 \pi x-\sin 2 \pi x, \\
b(t) & =\exp (t), r(t)=(1+2 \pi) \exp (t), x \in[0,1], t \in[0,1] .
\end{aligned}
$$

It is easy to check that the conditions $\left(\widetilde{A}_{1}\right)-\left(\widetilde{A}_{4}\right)$ are satisfied. Then according to Theorem 3.1 the solution of the inverse problem exists and unique. In fact, it can easily be checked by direct substitution that the analytical solution is given by

$$
\{a(t), v(x, t)\}=\{1 / \exp (t),(1+2 \pi x-\sin 2 \pi x) \exp (t)\} .
$$

Figure 4.1 shows the exact and numerical solution of $\{a(t), v(x, t)\}$ for $n t=128$ and $n x=64$. Next, we investigate the stability of numerical solution with respect to the noisy over-determination data (1.4), denoted by the function $r_{\gamma}(t)=r(t)(1+\gamma \theta)$ where $\gamma$ is the percentage of noise and $\theta$ are random variables generated from a uniform distribution in the interval $[-0.5,0.5]$ which are generated using rand command in MATLAB. Figs. 4.2, 4.3 show the exact and numerical solutions of $\{a(t), v(n x / 2, t)\}$ when the input data (1.4) is contaminated by $\gamma=1 \%, 3 \%$ and $5 \%$ noise. Figs. 4.4, 4.5 show the exact and numerical solutions of $\{a(t), v(n x / 2, t)\}$ obtained after mollification, when the input data (1.4) is contaminated by $\gamma=1 \%, 3 \%$ and $5 \%$ noise. This mollification procedure has been performed using MATLAB version of the computational program supplied by D. A. Murio in [13]. From these figures it can be seen that the application of the mollification to stabilize the noisy function $r_{\gamma}(t)$, produce stable numerical solutions for $\{a(t), v(n x / 2, t)\}$.
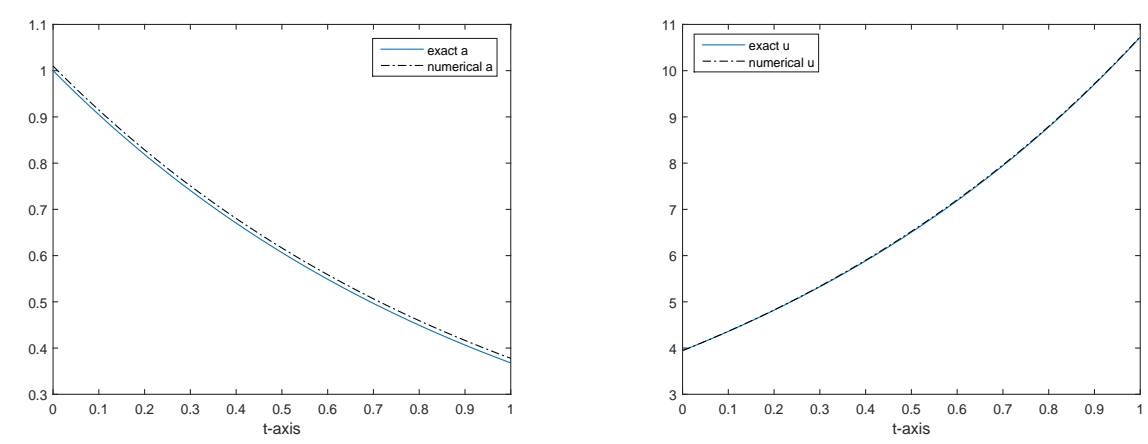

Figure 4.1: Exact and numerical solution of the problem (1)-(4) for example 4.1.

\section{Conclusion}

The inverse problems for linear wave equations connected with recovery of the coefficient are scarce. The paper considers the inverse problem of recovering a time-dependent coefficient in an initial boundary value problem for a wave equation with a non-homogeneous boundary condition. The series expansion method in terms of eigenfunctions of a Sturm-Liouville problem converts the considered inverse problem to a fixed point problem in a suitable Banach space. Under some conditions on the data, the existence and uniqueness of inverse problem is shown by using the Banach fixed point theorem. Numerically, the inverse problem has been discretized by using finite difference method, which has been solved using the MATLAB. Numerical results show that accurate, and stable solutions have been obtained. 

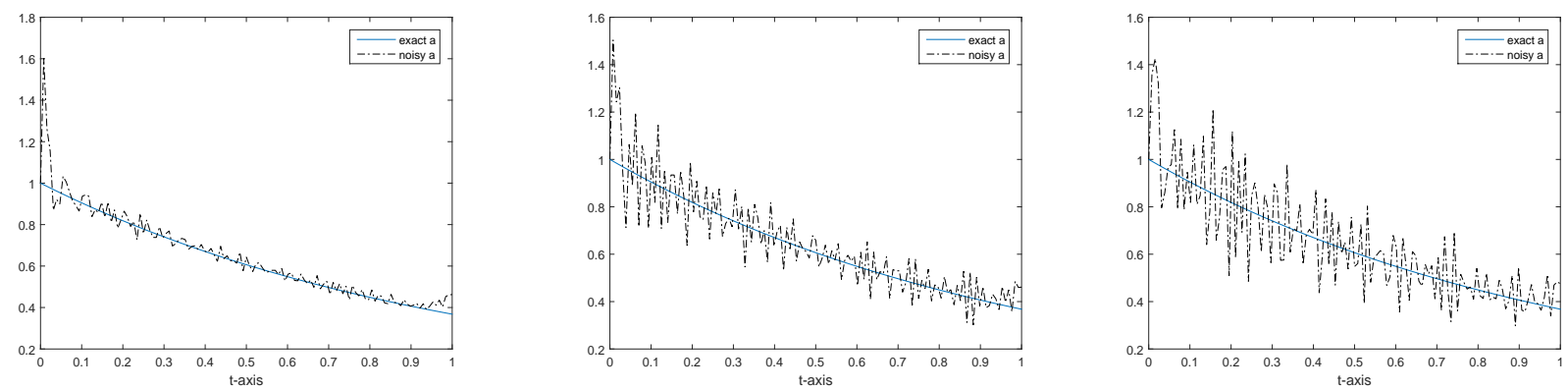

Figure 4.2: Exact and numerical coefficient solution of the problem (1)-(4) for example 4.1 with $1 \%, 3 \%$ and $5 \%$ noise.
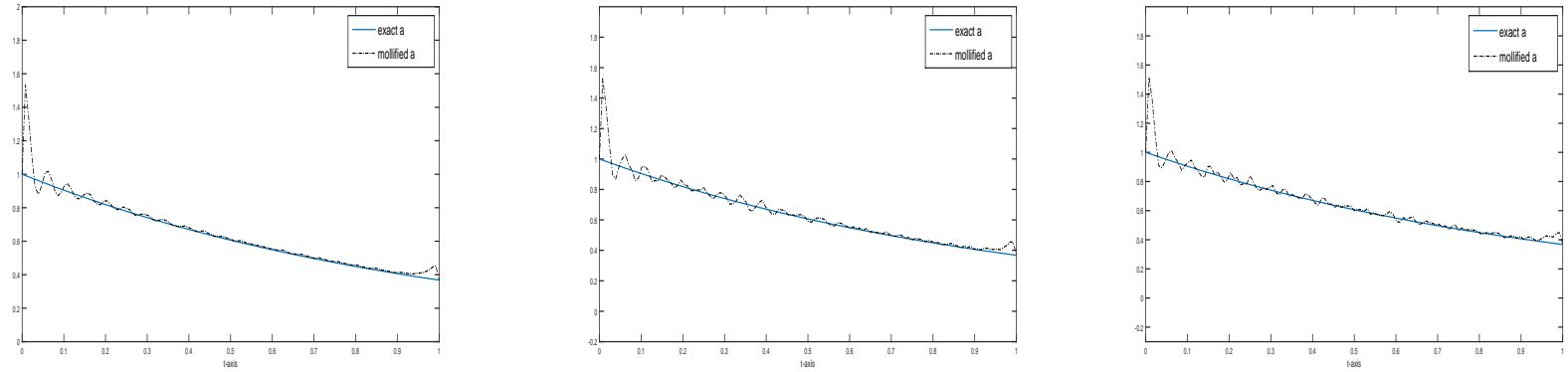

Figure 4.3: Exact and numerical coefficient solution of the problem (1)-(4) for example 4.1 after mollification with $1 \%, 3 \%$ and 5\% noise.

\section{References}

[1] L. Beilina, M. V. Klibanov, "A globally convergent numerical method for a coefficient inverse problem.” SIAM Journal on Scientific Computing 31.1 (2008): 478-509.

[2] J. R. Cannon, P. DuChateau, "An inverse problem for an unknown source term in a wave equation." SIAM Journal on Applied Mathematics 43.3 (1983): 553-564.

[3] M. Dehghan, "On the solution of an initial-boundary value problem that combines Neumann and integral condition for the wave equation." Numerical Methods for Partial Differential Equations 21.1 (2005): 24-40.

[4] S. O. Hussein, D. Lesnic, M. Yamamoto, "Reconstruction of space-dependent potential and/or damping coefficients in the wave equation." Computers \& Mathematics with Applications 74.6 (2017): 1435-1454.

[5] O. Imanuvilov, M. Yamamoto, "Global uniqueness and stability in determining coefficients of wave equations." Comm. Part. Diff. Equat., 26 (2001), $1409-1425$.

[6] N. I. Ionkin, "The solution of a certain boundary value problem of the theory of heat conduction with a nonclassical boundary condition." Differ. Uravn., 1977, Volume 13, Number 2, 294-304

[7] V. Isakov, Inverse problems for partial differential equations. Applied mathematical sciences. New York (NY): Springer; 2006.

[8] K. I. Khudaverdiyev, A. G. Alieva, "On the global existence of solution to one-dimensional fourth order nonlinear Sobolev type equations." Appl. Math. Comput. 217 (2010), no. 1, 347-354.

[9] D. Lesnic, S. O. Hussein, B. T. Johansson, "Inverse space-dependent force problems for the wave equation." Journal of Computational and Applied Mathematics 306 (2016): 10-39.

[10] Z. Lin, R. P. Gilbert, "Numerical algorithm based on transmutation for solving inverse wave equation.” Mathematical and computer modelling 39.13 (2004): 1467-1476.

[11] Y. Megraliev, Q. N. Isgenderova, "Inverse boundary value problem for a second-order hyperbolic equation with integral condition of the first kind." Problemy Fiziki, Matematiki i Tekhniki (Problems of Physics, Mathematics and Technics) 1 (2016): 42-47.

[12] Y. T. Mehraliyev, "On the identification of a linear source for the second order elliptic equation with integral condition.” Tr. Inst. Mat., 2013, Volume 21, Number 2, 128-141

[13] D.A. Murio, Mollification and space marching, in: K.A.Woodbury (Ed.), Inverse Engineering Handbook, CRC Press, Boca Raton, Florida, 2002, pp.

[14] G. K. Namazov, Inverse Problems of the Theory of Equations of Mathematical Physics, Baku, Azerbaijan, 1984. (in Russian).

[15] A. I. Prilepko, D. G. Orlovsky, I. A. Vasin, Methods for solving inverse problems in mathematical physics. Vol. 231, Pure and AppliedMathematics. New York (NY): Marcel Dekker; 2000.

[16] V.G. Romanov, Inverse Problems of Mathematical Physics, VNU Science Press BV, Utrecht, Netherlands, 1987.

[17] K. Šišková, M. Slodička, "Recognition of a time-dependent source in a time-fractional wave equation.” Applied Numerical Mathematics 121 (2017): $1-17$. 

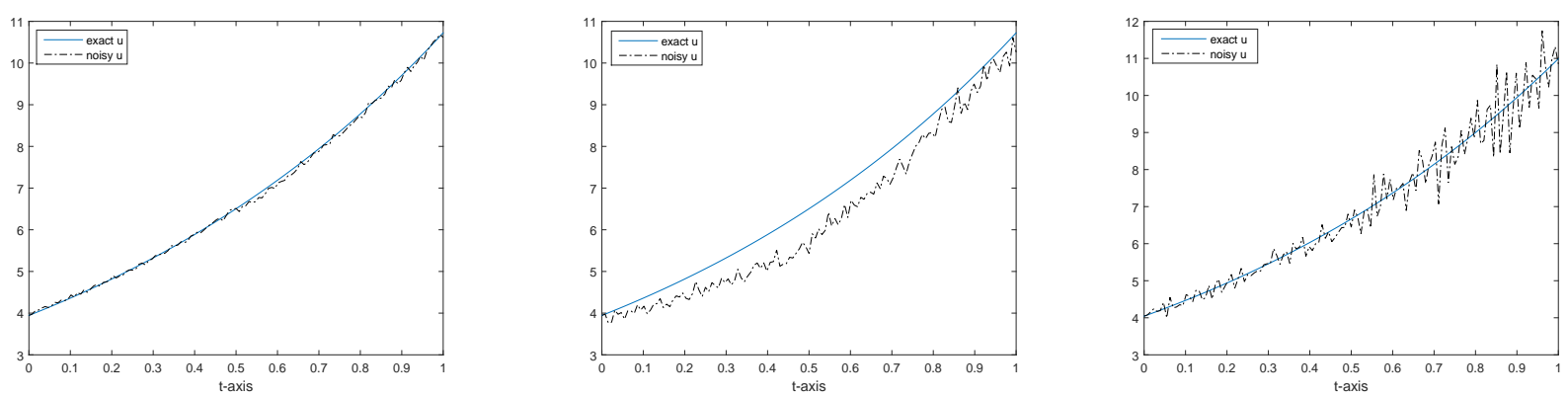

Figure 4.4: Exact and numerical $u(x, t)$ solution of the problem (1)-(4) for example 1 with $1 \%, 3 \%$ and $5 \%$ noise.
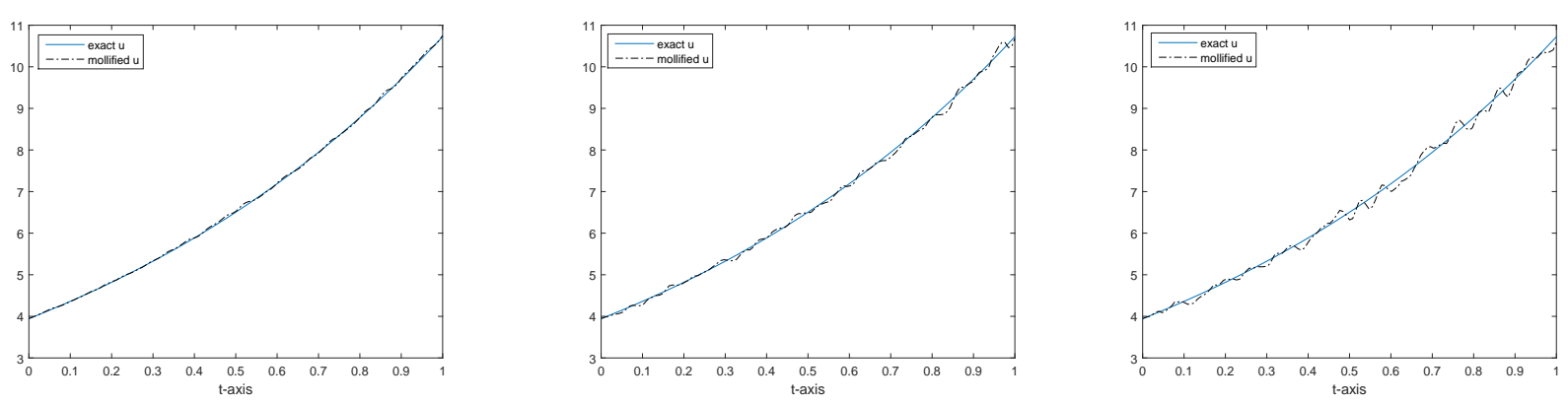

Figure 4.5: Exact and numerical $u(x, t)$ solution of the problem (1)-(4) for example 1 after mollification with $1 \%, 3 \%$ and $5 \%$ noise. 